\title{
GuavaH: a compendium of host genomic data in HIV biology and disease
}

István Bartha ${ }^{1,2,3}$, Paul J McLaren ${ }^{1,2,3}$, Angela Ciuffi ${ }^{1}$, Jacques Fellay ${ }^{1,2,3^{*}}$ and Amalio Telenti ${ }^{{ }^{*}}$

\begin{abstract}
Background: There is an ever-increasing volume of data on host genes that are modulated during HIV infection, influence disease susceptibility or carry genetic variants that impact HIV infection. We created GuavaH (Genomic Utility for Association and Viral Analyses in HIV, http://www.GuavaH.org), a public resource that supports multipurpose analysis of genome-wide genetic variation and gene expression profile across multiple phenotypes relevant to HIV biology.

Findings: We included original data from 8 genome and transcriptome studies addressing viral and host responses in and ex vivo. These studies cover phenotypes such as HIV acquisition, plasma viral load, disease progression, viral replication cycle, latency and viral-host genome interaction. This represents genome-wide association data from more than 4,000 individuals, exome sequencing data from 392 individuals, in vivo transcriptome microarray data from 127 patients/conditions, and 60 sets of RNA-seq data. Additionally, GuavaH allows visualization of protein variation in $~ 8,000$ individuals from the general population. The publicly available GuavaH framework supports queries on (i) unique single nucleotide polymorphism across different HIV related phenotypes, (ii) gene structure and variation, (iii) in vivo gene expression in the setting of human infection (CD4+ T cells), and (iv) in vitro gene expression data in models of permissive infection, latency and reactivation.

Conclusions: The complexity of the analysis of host genetic influences on HIV biology and pathogenesis calls for comprehensive motors of research on curated data. The tool developed here allows queries and supports validation of the rapidly growing body of host genomic information pertinent to HIV research.
\end{abstract}

Keywords: Genome-wide association studies, Exome analysis, Transcriptome analysis, HIV latency

\section{Findings}

The field of HIV research has adopted genome-wide technologies in order to meet the goal of understanding the complex interplay between host and pathogen. A growing number of approaches allow the interrogation of DNA variation (genome-wide genotyping, exome and whole genome sequencing), RNA variation (transcriptome analyses by gene expression arrays or deep sequencing), as well as large-scale functional screens (gene silencing using siRNA or shRNA, gain of function using gene overexpression). This is complemented with proteome and protein interaction analyses. The objective of these studies is to

\footnotetext{
*Correspondence: Jacques.fellay@epfl.ch; Amalio.telenti@chuv.ch ${ }^{1}$ Institute of Microbiology, University Hospital Lausanne, Lausanne, Switzerland ${ }^{2}$ School of Life Sciences, École Polytechnique Fédérale de Lausanne, Lausanne, Switzerland

Full list of author information is available at the end of the article
}

characterize the behavior of any gene/protein in the context of HIV infection in vitro or in vivo.

These studies are generally evaluated using strict statistics, which are necessary considering the large number of hypotheses that are simultaneously tested in most genome-wide scans. In addition, many studies require external validation, such as association results in a separate set of infected individuals, or expression results across various biological conditions. Accessing those resources is complex because raw data, or complete sets of analysis statistics are rarely available - or require re-contacting the original sources. Currently, there is a lack of integrated analysis tools by which researchers can easily access well curated data; to reinforce their own observations, for external replication or for generation of novel hypotheses.

Our groups have been involved in the generation and analysis of multiple such large-scale datasets. Thus, we aimed at building a simple platform that would facilitate 
the comparison of genomic and transcriptomic results across studies, while preserving the scientific interests of researchers and the privacy of study participants. This paper describes the structure of GuavaH (Genomic Utility for Association and Viral Analyses in HIV, http://www. GuavaH.org) and the central issues of interpretation and integration of genome-wide association (GWAS), exome and transcriptome data generated in the context of HIV research (Figure 1).

GuavaH currently provides results from GWAS of HIV disease phenotypes including more than 4,000 individuals. GWAS use large-scale genotyping technology (usually arrays interrogating 500,000 to 1 million single nucleotide polymorphisms, SNPs) complemented with statistical approaches that allow imputation of millions of additional variants that are not directly measured by the assay. The main challenge of GWAS is the stringent statistical threshold for claiming association (usually $\mathrm{p}<5 \times 10^{-8}$ ). The power to identify SNPs associated with a given phenotype depends on the frequency and the effect size of the genetic variant, and on sample size. Thus, large numbers of study participants and metaanalyses across studies are required. GuavaH includes association results on HIV control (set point plasma viral load [1,2] and elite control [3]) and on susceptibility to infection in a cohort of highly exposed seronegative individuals [4]. In addition to these traditional GWAS of clinically related outcomes GuavaH includes data from a recent genome-to-genome analysis of host genetic variants impacting the nucleic acid sequence of the infecting virus [5]. The genome-to-genome approach identifies loci of host-pathogen conflict independently of clinical data. Thus, GuavaH allows the interrogation of any SNP across multiple studies and phenotypes, and facilitates the validation of associations identified in other studies.

Large amounts of biological and genomic data are generated by additional emerging technologies. One approach that is transforming genome analysis is the study of human exome variation by high-throughput sequencing. In contrast to genotyping arrays, which only capture relatively common variation, exome sequencing captures all variants present in the coding regions of the genome: common, rare, and private. Each individual harbors about 20,000 unique coding variants, including a number of potentially severe functional variants coding for stop codons

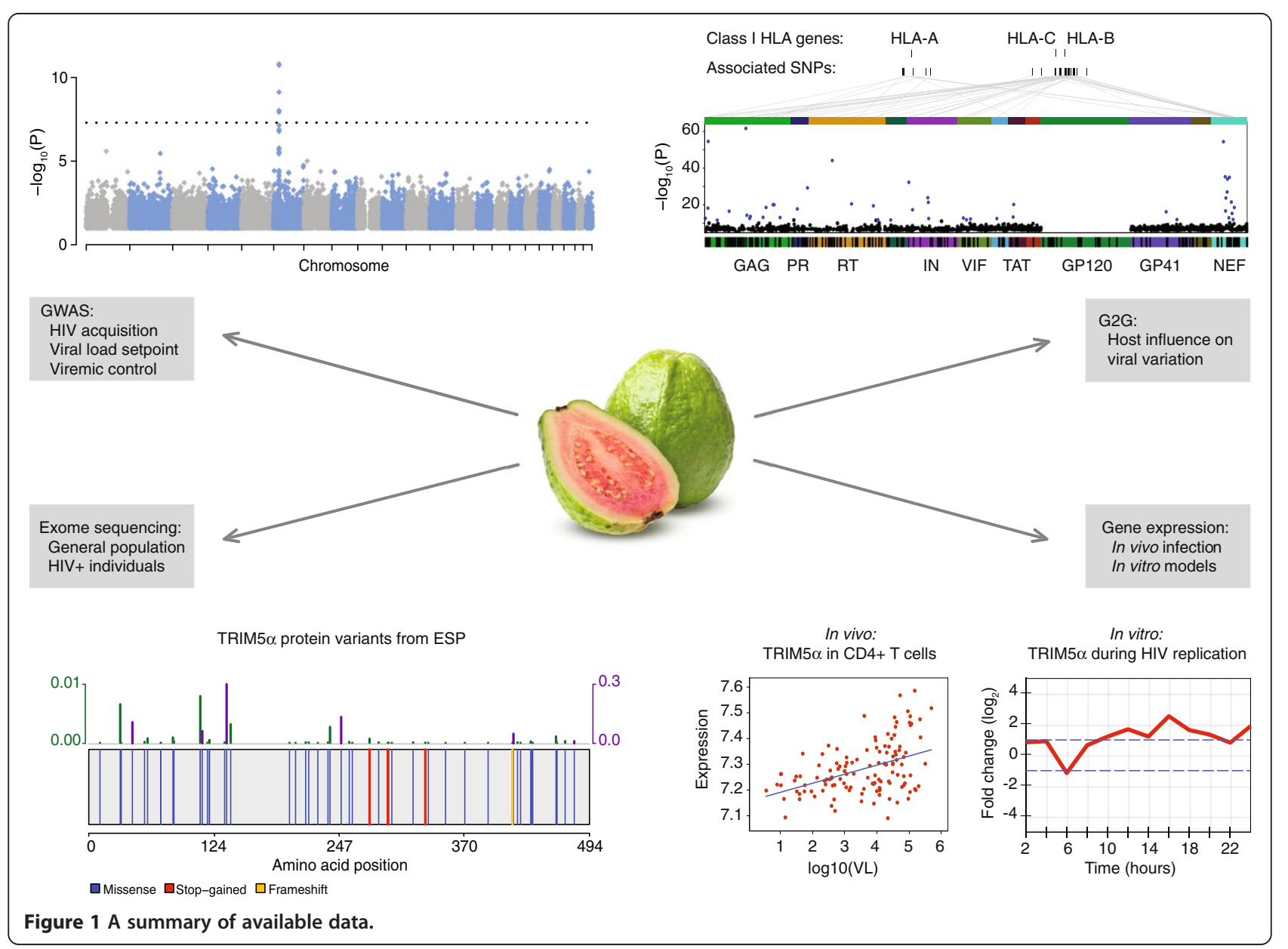


and for frameshift insertions or deletions [6]. Analyses are still complex, as there are statistical and functional limitations to the interpretation of rare variants. GuavaH provides gene-level/regional p-values and a graphical representation of nonsynonymous variants, premature stop codons and frameshift variants. We include protein-level sequence variation on a large sample taken from the general population (Analysis of more than 8000 exomes from the Exome Sequencing Project (http://evs.gs.washington. edu/EVS/)), and on 392 HIV infected individuals. Access to exome data in the HIV + sample is restricted due to data protection requirements, but gene-level queries are possible upon request (contact@guavah.org). This detailed level of protein sequence variation information allows for visualization and first-pass estimation of the mutational burden of a given gene (i.e. level of conservation or variation) and provides easy access to the genomic location and impact of protein variants in human genes that may be of importance in the HIV life cycle. For example, Figures 1 and 2 present the exome structure of TRIM5 $\alpha$ and CCR5, respectively. For both genes, the report identifies a number of rare premature stop codons.

The GuavaH resource also includes functional transcriptome analyses from in vivo and in vitro studies. The in vivo data were obtained by microarray studies of $\mathrm{CD} 4+\mathrm{T}$ cells from 127 individuals chronically infected with HIV, and representing the full spectrum of viral load [7]. These data can be contrasted with temporal in vitro analysis of the HIV replication cycle in a T cell line (Sup T1), representing 12 data points from HIV infected cells and 12 data points

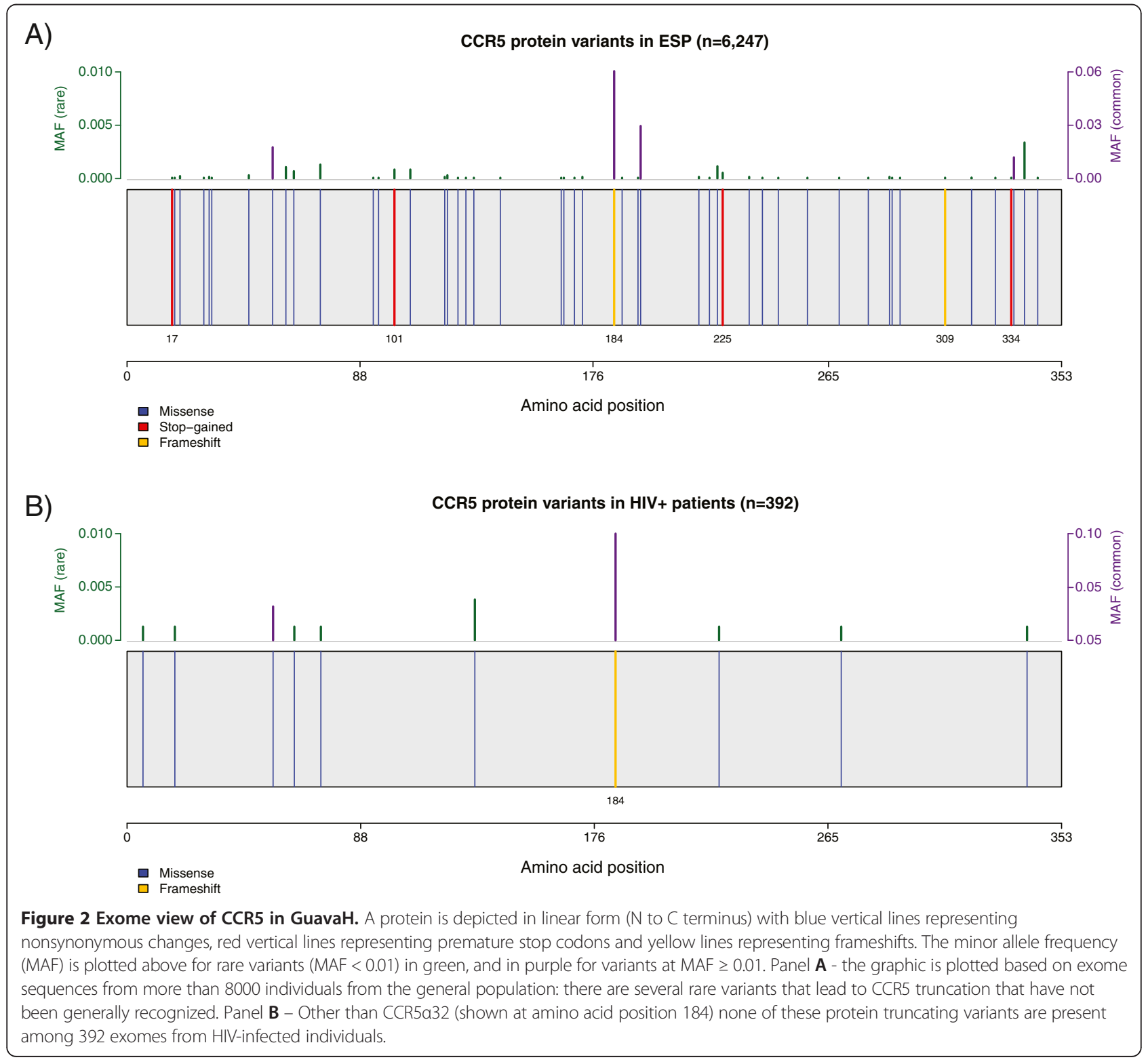


Table 1 Online resources on host genes in HIV biology and disease

\begin{tabular}{|c|c|c|}
\hline Web site & URL & Content \\
\hline \multicolumn{3}{|l|}{ Associated sites to GuavaH } \\
\hline PEACHi & http://peachi.labtelenti.org & Querying of cellular responses to HIV in vitro (SupT1 cells) \\
\hline LITCHi & http://litchi.labtelenti.org & $\begin{array}{l}\text { Querying of expression data during HIV latency and upon reactivation in } \\
\text { a primary CD4+ T cell model }\end{array}$ \\
\hline G2G & http://g2g.labtelenti.org & $\begin{array}{l}\text { Interactive HIV-host genome-to-genome map of the HLA class I locus } \\
\text { and viral genome variation }\end{array}$ \\
\hline \multicolumn{3}{|l|}{ External sites } \\
\hline Gene overlapper & $\begin{array}{l}\text { http://hivsystemsbiology.org/ } \\
\text { GeneListOverlapper/ }\end{array}$ & $\begin{array}{l}\text { Interactive overlapping of output from genome-wide surveys of host cell } \\
\text { genes linked to HIV infection }\end{array}$ \\
\hline $\begin{array}{l}\text { NCBI HIV-1 Human protein } \\
\text { interaction database }\end{array}$ & $\begin{array}{l}\text { http://www.ncbi.nlm.nih.gov/projects/ } \\
\text { RefSeq/HIVInteractions/ }\end{array}$ & The HIV-1, human protein interaction data are based on literature reports. \\
\hline Reactome HIV & http://reactome.org & Visualization, interpretation and analysis of pathway knowledge \\
\hline $\begin{array}{l}\text { VirusMINT - Virus molecular } \\
\text { interaction database }\end{array}$ & $\begin{array}{l}\text { http://mint.bio.uniroma2.it/virusmint/ } \\
\text { Welcome.do }\end{array}$ & $\begin{array}{l}\text { Interactions between human and HIV proteins are integrated in the } \\
\text { human protein interaction network }\end{array}$ \\
\hline
\end{tabular}

from uninfected cells analysed by sequencing [8]. For example, Figure 1 illustrates the in vivo and in vitro increase in TRIM5 $\alpha$ expression during active HIV-1 infection. Given the growing importance of latency research, we also incorporated detailed RNA sequencing data on the dynamic process of entering and maintaining latency in a primary cell model, and on the expression changes in host and viral transcripts upon reactivation with various pharmacological agents and immunological stimuli. GuavaH allows the interrogation of any gene across studies and cellular systems, and facilitates the validation of expression profiles identified in other studies.

GuavaH does not report on some additional large-scale genome-wide data (siRNA, gain-of-function screens) or on HIV-host protein interactions because these data are conveniently available through other open access resources (see [9] and (Table 1)). GuavaH is also linked to other associated resources from our group that allow more detailed and interactive exploration of the genome-to-genome data, of the viral replication cycle dynamics, and on the latency models (Table 1). Expected additions to GuavaH in coming months are proteome and phosphoproteome data, and additional transcriptome datasets from primary cell models of latency.

Promoting easy access to genome-wide association and functional data fits the goal defined in 2009 by The Global HIV Vaccine Enterprise of understanding the role of host genetics in HIV research: "New high-throughput genetic approaches have the potential to identify major genetic factors contributing to clinical outcome in HIV-1 infection. Ideally, every human gene that impacts on each mode of HIV transmission and disease outcome should be identified to improve our understanding of the mechanisms of protection" [10]. GuavaH is a useful tool for visualizing the host genomic effects attributable to a given gene of interest and its potential functional implications in a variety of in vitro and in vivo settings of HIV infection.

\section{Availability of supporting data}

GuavaH provides access to published datasets and to unpublished data upon discussion with the researchers in charge of the original work. It also allows depositing of new sets of data for public or private querying. Contact: contact@guavah.org

\section{Competing interests}

The authors declare that they have no competing interests. GuavaH is an academic initiative supported with funds from the Swiss National Science Foundation.

\section{Authors' contributions}

IB developed the web interface, and is responsible for generation of the genome-to-genome data, PM is primarily responsible for generation and curation of genome wide association data, AC is responsible for generation and curation of expression data, JF and AT designed and executed the original studies. All authors contributed to the manuscript and final design of the web interface. All authors read and approved the final manuscript.

\section{Acknowledgements}

Paul de Bakker, Florencia Pereyra, Bruce Walker, David Goldstein, Pejman Mohammadi, Julia di Iulio and Margalida Rotger for their contributions to the original data presented in this website.

\section{Author details}

${ }^{1}$ Institute of Microbiology, University Hospital Lausanne, Lausanne, Switzerland.

${ }^{2}$ School of Life Sciences, École Polytechnique Fédérale de Lausanne, Lausanne,

Switzerland. ${ }^{3}$ Swiss Institute of Bioinformatics, Lausanne, Switzerland.

Received: 3 December 2013 Accepted: 7 January 2014

Published: 15 January 2014

\section{References}

1. Fellay J, Ge D, Shianna KV, Colombo S, Ledergerber B, Cirulli ET, Urban TJ, Zhang K, Gumbs CE, Smith JP, et al: Common genetic variation and the control of HIV-1 in humans. PLoS Genet 2009, 5:e1000791.

2. Fellay J, Shianna KV, Ge D, Colombo S, Ledergerber B, Weale M, Zhang K, Gumbs C, Castagna A, Cossarizza A, et al: A whole-genome association study of major determinants for host control of HIV-1. Science 2007, 317:944-947. 
3. International HIVCS, Pereyra F, Jia X, McLaren PJ, Telenti A, de Bakker PI, Walker BD, Ripke S, Brumme CJ, Pulit SL, et al: The major genetic determinants of HIV-1 control affect HLA class I peptide presentation. Science 2010, 330:1551-1557.

4. Lane J, McLaren PJ, Dorrell L, Shianna KV, Stemke A, Pelak K, Moore S, Oldenburg J, Alvarez-Roman MT, Angelillo-Scherrer A, et al: A genome-wide association study of resistance to HIV infection in highly exposed uninfected individuals with hemophilia A. Hum Mol Genet 2013, 22:1903-1910.

5. Bartha I, Carlson JM, Brumme CJ, McLaren PJ, Brumme ZL, John M, Haas DW, Martinez-Picado J, Dalmau J, Lopez-Galindez C, et al: A genome-togenome analysis of associations between human genetic variation, HIV-1 sequence diversity, and viral control. elife 2013, 2:e01123.

6. MacArthur DG, Balasubramanian S, Frankish A, Huang N, Morris J, Walter K, Jostins L, Habegger L, Pickrell JK, Montgomery SB, et al: A systematic survey of loss-of-function variants in human protein-coding genes. Science 2012, 335:823-828.

7. Rotger M, Dang KK, Fellay J, Heinzen EL, Feng S, Descombes P, Shianna KV Ge D, Gunthard HF, Goldstein DB, et al: Genome-wide mRNA expression correlates of viral control in CD4+ T-cells from HIV-1-infected individuals. PLoS Pathog 2010, 6:e1000781.

8. Mohammadi P, Desfarges S, Bartha I, Joos B, Zangger N, Munoz M, Gunthard HF, Beerenwinkel N, Telenti A, Ciuffi A: 24 hours in the life of HIV-1 in a T cell line. PLoS Pathog 2013, 9:e1003161.

9. Bushman FD, Barton S, Bailey A, Greig C, Malani N, Bandyopadhyay S, Young J, Chanda S, Krogan N: Bringing it all together: big data and HIV research. Aids 2013, 27:835-838.

10. McMichael AJ, McCutchan F: Host genetics and viral diversity: report from a global HIV vaccine enterprise working group. Nat Prec 2010. doi:10.1038/npre.2010.4797.2.

doi:10.1186/1742-4690-11-6

Cite this article as: Bartha et al: GuavaH: a compendium of host genomic data in HIV biology and disease. Retrovirology 2014 11:6.

\section{Submit your next manuscript to BioMed Central and take full advantage of:}

- Convenient online submission

- Thorough peer review

- No space constraints or color figure charges

- Immediate publication on acceptance

- Inclusion in PubMed, CAS, Scopus and Google Scholar

- Research which is freely available for redistribution

Submit your manuscript at www.biomedcentral.com/submit
C Biomed Central 